\title{
BRIÓFITAS DE CAATINGA. 2. AGRESTINA, PERNAMBUCO, BRASIL
}

\author{
Kátia Cavalcanti Pôrto ${ }^{1}$ \\ Maria de Fátima de Andrade Bezerra ${ }^{2}$
}

Recebido em 30.06.94. Aceito em 09.03.96.

RESUMO - (Briófitas de caatinga 2. Agrestina, Pernambuco, Brasil). Em uma área de caatinga do município de Agrestina (Lat. 8²7'28”'S; Long. 3557'13”W) - PE foram identificadas 13 espécies de Bryophyta, pertencentes as famílias Frullaniaceae, Lejeuneaceae, Ricciaceae - Hepaticopsida Bryaceae, Dicranaceae, Ephemeraceae, Fissidentaceae, Leucobryaceae, Pottiaceae e Stereophyllaceae - Bryopsida. Constituem-se novos registros para a região Nordeste Cololejeunea minutissima (Sm.) Schiffn. e Riccia brasiliensis Schiffn. e para Pernambuco Frullania dusenii Steph.

Palavras-chave: Bryophyta, caatinga, Pernambuco.

\begin{abstract}
Bryophytes of caatinga 2. Agrestina, Pernambuco, Brasil). In a caatinga area,

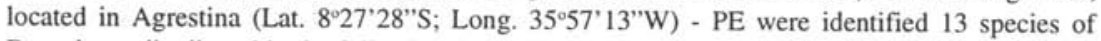
Bryophyte, distributed in the following families Frullaniaceae, Lejeuneaceae, Ricciaceae - Hepaticopsida - Bryaceae, Dicranaceae, Ephemeraceae, Fissidentaceae, Leucobryaceae, Pottiaceae e Stereophyllaceae - Bryopsida. Cololejeunea minutissima (Sm.) Schiffn. and Riccia brasiliensis Schiffn. are new records for the Northeast region, while Frullania dusenii Steph. represents a new record for the Pernambuco State.
\end{abstract}

Key words: bryophyte, caatinga, Pernambuco.

\section{Introdução}

Em ambientes semi-áridos do Nordeste brasileiro é evidente a escassez de publicações sobre a ocorrência de briófitas. No que concerne o Estado de Pernambuco, destacam-se os trabalhos de Yano \& Andrade-Lima (1987), que levantaram as briófitas de diversas áreas úmidas enclavadas na caatinga - regionalmente denominadas de brejos - e os de Pôrto (1990), que relacionou 107 espécies para Brejo dos Cavalos, em Caruaru. Os autores acima referidos e Pôrto (1992) foram unânimes em destacar a supreendente

\footnotetext{
${ }^{1}$ Departamento de Botânica, CCB, UFPE. 50670-710, Recife - PE.

2 Bolsista de Aperfeiçoamento, CNPq, Processo n ${ }^{\circ} 824504 / 90-8$.
} 
riqueza da flora briofítica dos brejos, certamente, bastante diversa das encontradas em locais mais baixos, de maior aridez. Recentemente, Pôrto et al. (1994) determinaram a brioflora de uma área de caatinga arbórea no município de Caruaru, registrando 17 espécies. Visando adicionar mais algumas informações acerca das briófitas ocorrentes neste tipo de formação vegetal, realizou-se o presente trabalho.

\section{Material e métodos}

A área de caatinga investigada pertence a Fazenda Riacho do Peixe, município

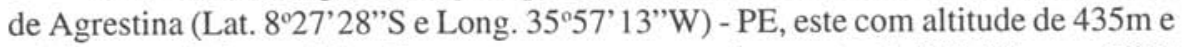
médias anuais de precipitação e temperatura, respectivamente, de $858,00 \mathrm{~mm}$ e $26^{\circ} \mathrm{C}$ (Dados fornecidos pela Superintendência de Desenvolvimento do Nordeste - SUDENE). O clima segundo Koeppen, é do tipo AS'- quente e úmido, com estação chuvosa concentrada de fevereiro a julho, iniciando-se em agosto a estação seca, que se prolonga até janeiro (Fig. 1).
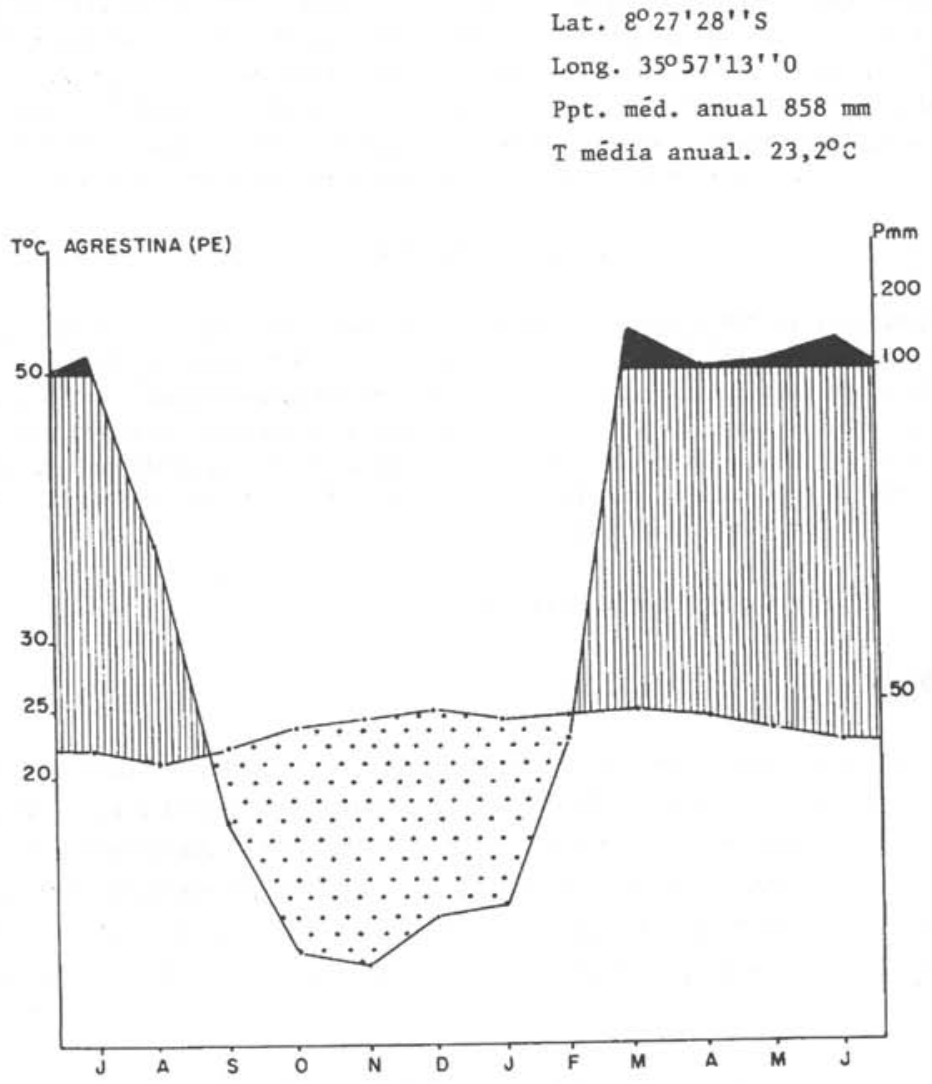

Figura 1. Diagrama ombro-térmico para o município de Agrestina-PE. 
A referida área tem aproximadamente 6 ha, sendo mantida livre de desmate e queima por parte dos proprietários. As fanerógamas de maior porte no local atingem $7 \mathrm{~m}$ de altura, sendo representadas, principalmente por Ziziphus joazeiro Mart., Spondias tuberosa Arr. Cam., Schinopsis brasiliensis Engl. e Mimosa spp.

As amostras de briófitas foram coletadas durante três excursões mensais, realizadas de maio a julho de 1993.

Para a identificação do material consultaram-se Bartram (1949), Florschütz (1964), Gradstein (1989), Griffin III (1979), Jovet-Ast (1991, 1993) e Yuzawa (1991) e exsicatas existentes no Herbário UFP, identificadas por especialistas.

Após estudo, as amostras foram depositadas no Herbário UFP, da Universidade Federal de Pernambuco.

\section{Resultados}

Na área de caatinga estudada identificaram-se 13 espécies de briófitas, sendo 5 pertencentes a Hepaticopsida e 8 a Bryopsida, distribuídas, respectivamente, em 3 e 7 famílias.

\section{HEPATICOPSIDA}

\section{FRULLANIACEAE}

Frullania dusenii Steph., Arch. Mus. Nat. Rio de Janeiro, 13:115. 1903.

Descrição e ilustração: Yuzawa (1991).

Material examinado: PE, Agrestina, Fazenda Riacho do Peixe, K.C. Pôrto 13/V/93, UFP 9131; K.C. Pôrto 13/V/93, UFP 9132; K.C. Pôrto 1/VI/93, UFP 9141; K.C. Pôrto 1/VI/93, UFP 9143; M.F.A. Bezerra 8/VII/93, UFP 9153; M.F.A. Bezerra 8/VII/93, UFP 9155.

Comentários: Corticícola, geralmente associada a Cololejeunea minutissima (Sm.) Schiffn.

São características importantes para o reconhecimento desta espécie os filídios orbiculares de lóbulo galeado e estilete reduzido, o anfigastro orbicular, curtamente bífido e o perianto tetra-quilhado.

Registrada para o Rio Grande do Sul, Rio de Janeiro (Yano 1984); Sergipe (Yano 1994), sendo esta a primeira citação para Pernambuco.

Frullania riojaneirensis (Raddi) Aongst., Ofvers. K. Vet-Akad. Forjandl. 7:88. 1876.

Descrição e ilustração: Yuzawa (1991). 
Material examinado: PE, Agrestina, Fazenda Riacho do Peixe, K.C. Pôrto 13/V/93, UFP 9135; K.C. Pôrto 1/VI/93, UFP 9143.

Comentários: Corticícola.

Espécie caracterizada pelo lóbulo galeado, o anfigastro de inserção cordiforme e as brácteas/bractéolas inteiras ou esparsamente denteadas; o perianto é fusiforme, tetra-quilhado, ou mais raramente pluriplicado (Haarbrink 1981).

Registrada para Goiás, Mato Grosso, Minas Gerais, Rio Grande do Sul, Rio de Janeiro, São Paulo (Yano 1984); Pernambuco (Pôrto 1990); Espírito Santo (SchäferVerwimp 1991); Bahia, Distrito Federal (Yuzawa 1991).

\section{LEJEUNEACEAE}

Cololejeunea minutissima (Sm.) Schiffn., Engler \& Prantl., Naturl. Pflanzenfam. 1(3):122. 1895.

Descrição e ilustração: Schuster (1955).

Material examinado: PE, Agrestina, Fazenda Riacho do Peixe, M.F.A. Bezerra 13/V/ 93, UFP 9128; K.C. Pôrto 8/VII/93, UFP 9151; K.C. Pôrto 8/VII/93, UFP 9156.

Comentários: Corticícola, associada a Frullania dusenii Steph.

Esta espécie monóica é reconhecida pelos filídios sub-orbiculares, de margens crenuladas e lóbulos grandes ou, às vezes reduzidos, com 2 dentes, o distal formado por 2 células. O material estudado apresentava numerosas gemas discoidais, de 24 células cada, dispostas na face ventral dos filídios e dos androecia.

Registrada para o Espírito Santo (Schäfer-Verwimp 1991); Roraima (Yano e Mello 1992); sendo este o primeiro registro para a região Nordeste.

\section{RICCIACEAE}

Riccia brasiliensis Schiffn., Osterr., Akad. Wiss. Math.-Naturwiss. Kl., Denkschr., 111:6. 1964.

Descrição e ilustração: Jovet-Ast (1991).

Material examinado: PE, Agrestina, Fazenda Riacho do Peixe, K.C. Pôrto 1/VI/93, UFP 9147.

Comentários: Terrícola, sobre solo úmido, associada a Riccia stenophylla Spruce.

Caracterizam esta espécie o talo de bordos convexos e os cílios esparsos, violáceos, dispostos nas margens e no ápice dos lobos. 
Registrada para o Paraná (Yano 1984); Rio Grande do Sul, Santa Catarina, São Paulo (Jovet-Ast 1991), sendo esta a primeira citação para a região Nordeste.

Riccia stenophylla Spruce, Bull. Soc. Bot. France 36:195.”1889”. 1890.

Descrição e ilustração: Jovet-Ast (1993).

Material examinado: PE, Agrestina, Fazenda Riacho do Peixe, K.C. Pôrto 1/VI/93, UFP 9147.

Comentários: Terrícola, sobre solo úmido, associada a Riccia brasiliensis Schiffn.

Espécie caracterizada pelos lobos estreitos, de no máximo 1,5 $\mathrm{mm}$ de largura, as escamas ventrais hialinas, a morfologia e a ornamentação dos esporos.

Registrada para o Rio Grande do Sul (Vianna 1981); Pernambuco (Pôrto 1990); Bahia, Espírito Santo, Goiás, Mato Grosso, Paraná, Rio de Janeiro, Santa Catarina, São Paulo (Jovet-Ast 1993).

\section{BRYOPSIDA}

\section{FISSIDENTACEAE}

Fissidens intermedius C. Muell., Linnaea 21:181.1848.

Descrição e ilustração: Florschütz (1964).

Material examinado: PE, Agrestina, Fazenda Riacho do Peixe, K.C. Pôrto 8/VII/93, UFP 9149a.

Comentários: Terrícola, sobre solo úmido.

Espécie com até 10 pares de filídios, estes de ápice agudo ou acuminado, com bordos restritos a lâmina vaginante; as células são, em geral, unipapilosas, embora células de paredes lisas também possam ocorrer.

Registrada para o Amazonas (Griffin III 1979), Pernambuco (Pôrto 1990); Rondônia (Lisboa 1991).

Fissidens pennula Broth., Bih. K. Vit. Ak. Handl. 26Afd. 3(7):13. 1900.

Descrição e ilustração: Florschütz (1964).

Material examinado: PE, Agrestina, Fazenda Riacho do Peixe, K.C. Pôrto 13/V/93, UFP 9134; K.C. Pôrto 13/V/93, UFP 9138; K.C. Pôrto 1/VI/93, UFP 9145; K.C. Pôrto 1/VI/93, UFP 9146; M.F.A. Bezerra 8/VII/93, UFP 9148. 
Comentários: Terrícola, sobre solo úmido e seco.

Espécie com até 25 pares de filídios, estes flexíveis, bordeados até o ápice e com células lisas.

Registrada para o Mato Grosso, Pará (Yano 1981); Pernambuco (Pôrto et al. 1994).

\section{DICRANACEAE}

Dicranella hilariana (Mont.) Mitt., Journ. Linn. Soc. London Bot. 12:31.1869.

Descrição e ilustração: Florschütz (1964).

Material examinado: PE, Agrestina, Fazenda Riacho do Peixe, K.C. Pôrto 1/VI/93, UFP 9139; K.C. Pôrto 8/VII/93, UFP 9154..

Comentários: Terrícola, sobre solo úmido, crescendo associada a Micromitrium thelephorothecum (Florsch.) Crosby.

Espécie de morfologia variável, mas facilmente reconhecida por apresentar filídios lanceolados, gradualmente acuminados, costa forte, percurrente até curtoexcurrente e células alares pouco diferenciadas.

Registrada para o Amazonas (Griffin III 1979); Bahia, Minas Gerais, Rio de Janeiro, Santa Catarina, São Paulo (Yano 1981); Paraíba (Marinho 1987); Pernambuco (Pôrto 1990); Rondônia (Lisboa \& Carreira 1990); Espírito Santo (SchäferVerwimp 1991); Roraima (Yano \& Mello 1992).

\section{POTTIACEAE}

Barbula agraria Hedw., Spec. Musc. 116.1801.

Descrição e ilustração: Florschütz (1964).

Material examinado: PE, Agrestina, Fazenda Riacho do Peixe, M.F.A. Bezerra 13/V/ 93, UFP 9130.

Comentários: Saxícola.

Espécie caracterizada por filídios oblongos, de ápice inteiro e acuminado, células pequenas e mamilosas e peristômio com dentes longos e espiralados.

Registrada para o Amazonas, Rio de Janeiro (Yano 1981); Paraíba (Marinho 1987); Pernambuco (Vital et al. 1991).

LEUCOBRYACEAE

Octoblepharum albidum Hedw., Spec. Musc. 50. 1801. 
Descrição e ilustração: Florschütz (1964).

Material examinado: PE, Agrestina, Fazenda Riacho do Peixe, M.F.A. Bezerra 13/V/ 93, UFP 9123; M.F.A. Bezerra 13/V/93, UFP 9129.

Comentários: Corticícola, usualmente presente desde a base dos troncos até as primeiras ramificações, epixíla e raramente terrícola.

Espécie de fácil reconhecimento apresentando filídios de ápice agudo ou obtuso, denteados, seta curta, $2-8 \mathrm{~mm}$ de comp. e peristômio com 8 dentes triangulares.

Registrada para o Amazonas, Bahia, Ceará, Espírito Santo, Goiás, Maranhão, Mato Grosso, Minas Gerais, Pará, Paraná, Pernambuco, Rio de Janeiro, Roraima, Santa Catarina, São Paulo (Yano 1981); Paraíba (Marinho 1987); Amapá, Rondônia (Yano 1989); Espírito Santo (Schäfer-Verwimp 1991); Acre, Alagoas, Distrito Federal, Mato Grosso do Sul, Piauí, Rio Grande do Norte, Rio Grande do Sul, Sergipe (Yano 1992).

\section{BRYACEAE}

Bryum coronatum Schwaegr., Spec. Musc. suppl. 1(2):193. 1816.

Descrição e ilustração: Florschütz (1964).

Material examinado: PE, Agrestina, Fazenda Riacho do Peixe, M.F.A. Bezerra 13/V/ 93, UFP 9124; M.F.A. Bezerra 13/V/93, UFP 9127; K.C. Pôrto 1/VI/93, UFP 9140; K.C. Pôrto 1/VI/93, UFP 9142; K.C. Pôrto 1/VI/93, UFP 9144; K.C. Pôrto 8/VII/93, UFP 9150; K.C. Pôrto 8/VII/93, UFP 9152.

Comentários: Corticícola, geralmente na base de troncos, epíxila e terrícola, eventualmente associada a Octoblepharum albidum Hedw.

Espécie variável, reconhecida principalmente pela seta cor vermelha até marrom escuro quando seca, cápsula pêndula e colo truncado.

Registrada para o Amazonas, Bahia, Goiás, Mato Grosso, Minas Gerais, Pará, Paraná, Rio Grande do Sul, Rio de Janeiro, São Paulo (Yano 1981); Paraíba (Marinho 1987); Pernambuco, Rondônia, Roraima, Santa Catarina (Yano 1989).

\section{EPHEMERACEAE}

Micromitrium thelephorothecum (Florsch.) Crosby, Bryologist 71:116. 1968.

Descrição e ilustração: Pôrto \& Yano (1985).

Material examinado: PE, Agrestina, Fazenda Riacho do Peixe, K.C. Pôrto 8/VII/93, UFP 9149b. 
Comentários: Terrícola, crescendo sobre o solo úmido, em associação com Fissidens intermedius C. Muell.

Espécie de fácil identificação, apesar do porte bastante reduzido, o que dificulta a sua visualização no campo. São características distintivas o protonema persistente, a cápsula globosa, gimnóstoma, com células mamilosas e esporos grandes, de até 65 um de diâmetro.

Registrada para Pernambuco (Pôrto \& Yano 1985); Rondônia (Lisboa 1991).

\section{STEREOPHYLLACEAE}

Entodontopsis leucostega (Brid.) Buck \& Irel., Nova Hedwigia 41:103. 1985.

Descrição e ilustração: Sharp et al. (1994).

Material examinado: PE, Agrestina, Fazenda Riacho do Peixe, M.F.A. Bezerra 13/V/ 93, UFP 9125; M.F.A. Bezerra 13/V/93, UFP 9126; M.F.A. Bezerra 13/V/93, UFP 9133; M.F.A. Bezerra 13/V/93, UFP 9136; M.F.A. Bezerra 13/V/93, UFP 9137; K.C. Pôrto 8/VII/93, UFP 9157.

Comentários: Corticícola e epíxila, geralmente associada a Octoblepharum albidum Hedw.

Caracteriza bem esta espécie as células alares quadráticas, numerosas, estendendo-se até a costa; os filídios acuminados, ligeiramente assimétricos; as células alongadas e as cápsulas pêndulas.

Registrada para o Amazonas, Bahia, Ceará, Goiás, Mato Grosso, Minas Gerais, Rio de Janeiro (Yano 1981); Pernambuco (Vital et al. 1991), São Paulo (Yano \& Santos 1993).

\section{Conclusões}

As citações de briófitas ocorrentes em áreas de caatinga são ainda bastante reduzidas. As escassas coletas realizadas nestes ambientes podem ser tanto uma resposta ao fato de que os briologistas raramente coletam nestas áreas, como a real escassez destas plantas, que quase nenhuma defesa possuem à dessecação. Contudo, algumas espécies mais resistentes, de ciclo de vida curto ou revivescentes podem ser encontradas em ambientes semi-áridos, como as caatingas.

$\mathrm{Na}$ área de caatinga estudada, pertencente ao município de Agrestina - PE, foram identificadas 13 espécies de briófitas, das quais 5 Hepaticopsida, das famílias Frullaniaceae (2 esp.), Lejeuneaceae e Ricciaceae (2 esp.) e 8 Bryopsida, das famílias Bryaceae, Dicranaceae, Ephemeraceae, Fissidentaceae (2 esp.), Leucobryaceae, Pottiaceae e Stereophyllaceae. Está sendo registrada pela primeira vez a ocorrência de Cololejeunea minutissima (Sm.) Schiffn. e Riccia brasiliensis Schiffn. para o Nordes- 
te brasileiro e de Frullania dusenii Steph. para o Estado de Pernambuco.

Os resultados obtidos indicam que os substratos de colonização predominantes para as amostras analisadas foram solo e troncos vivos, enquanto que troncos mortos e rochas são mais raramente utilizados.

\section{Agradecimentos}

Aos Drs. Y. Yuzawa, do Hattori Botanical Laboratory - Japão, S. Jovet-Ast e P. Tixier, ambos do Muséum National d'Histoire Naturelle - França, que gentilmente confirmaram as identificações de Frullania, Riccia e Cololejeunea, respectivamente.

\section{Referências bibliográficas}

Bartram, E.B. 1949. Mosses of Guatemala. Fieldiana, Bot., 25:1-442.

Florschütz, P.A. 1964. The mosses of Suriname. Leiden: E.J. Brill.

Gradstein, S.R. 1989. A key to the Hepaticae and Anthocerotae of Puerto Rico and Virgin Islands. Bryologist, 92(3):329-348.

Griffin III, D. 1979. Guia preliminar para as Briófitas freqüentes em Manaus e adjacências. Acta Amazonica, supl. 9(3):1-67.

Haarbrink, J. 1981. Studies on Colombian Cryptogams XI. High Andean species of Frullania subg. Chonanthelia (Hepaticae). Lindbergia, 7:47-57.

Jovet-Ast, S. 1991. Riccia (Hépatiques, Marchantiales) d'Amérique Latine. Taxons du sous-genre Riccia. Cryptogamie, Bryol. Lichénol., 12(3):189-370.

Jovet-Ast, 1993. Riccia L. (Hépatiques, Marchantiales) d'Amérique Latine. Taxons des sous-genres Thallocarpus, Leptoriccia, Ricciella. Cryptogamie, Bryol. Lichénol., 14(3):219-301.

Lisboa, R.C.L. 1991. Musgos acrocárpicos do Estado de Rondônia. São Paulo: Universidade de São Paulo. Tese de Doutorado.

Lisboa, R.C.L. \& Carreira, L.M.M. 1990. Contribuição ao conhecimento da flora do município de Guajará-Mirim (RO). In: Anais XXXV Congresso Nacional de Botânica. Anais... Brasilia, IBAMA. p.67-78.

Marinho, M.G.V. 1987. Bryopsida da reserva florestal do IBDF, João Pessoa, Paraiba, Brasil. Recife: Universidade Federal de Pernambuco. Dissertação de Mestrado.

Pôrto, K.C. 1990. Bryoflores d'une forêt de plaine et d'une forêt d'altitude moyenne dans l'Etat de Pernambuco (Brésil): analyse floristique. Cryptogamie, Bryol. Lichénol., 11(2):109-161.

Pôrto, K.C. 1992. Bryoflores d'une forêt de plaine et d'une forêt d'altitude moyenne dans l'Etat de Pernambuco (Brésil). 2. Analyse écologique comparative des forêts. Cryptogamie, Bryol, Lichénol., 13(3):187-219.

Pôrto, K.C. \& Yano, O. 1985. Novas ocorrências de Briófitas em Pernambuco. Rickia, 12:9-14.

Pôrto, K.C.; Silveira, M² F. G. \& SÁ, P.S.A. 1994. Briófitas de caatinga. 1. Estação Experimental do IPA. Caruaru - PE. Acta Botanica Brasilica, 8(1):77-85.

Schäfer-Verwimp, A. 1991. Contribution to the knowledge of the bryophyte flora of Espírito Santo, Brazil. Journ. Hattori Bot. Lab., 69:147-170.

Schuster, R.M. 1955. North American Lejeuneaceae III. Paradoxae: Cololejeunea, sectio Minutissimae. Journ. Elisha Mitchell Sci. Soc., 71(2):218-247.

Sharp, A.J.; Crum, H. \& Eckel, P.M. 1994. The Mosses flora of Mexico. Memoirs New York Botanical Garden, 69(1-2):1-1113.

Vianna, E. C. 1981. O gênero Riccia (Marchantiales) no Rio Grande do Sul, Brasil. Subgen. Ricciella e Thallocarpus. Rickia, 9:71-80.

Vital, D.; Giancotti, C. \& Pursell, R.A. 1991. The Bryoflora of Fernando de Noronha, Brazil. Tropical Bryology, 4:23-24. 
Yano, O. 1981. A checklist of Brazilian mosses. Journ. Hattori Bot. Lab., 50:279-456.

Yano, O. 1984. Checklist of Brazilian Liverworts and Hornworts. Journ. Hattori Bot. Lab., 56:481-548. Yano, O. 1989. An additional checklist of Brazilian bryophytes. Journ. Hattori Bot. Lab., 66:371-434. Yano, O. 1992. Leucobryaceae (Bryopsida) do Brasil. São Paulo: Universidade de São Paulo. Tese de Doutorado.

Yano, O. 1994. Briófitas da Serra de Itabaiana, Sergipe, Brasil. Acta Bot. Bras., 8(1):45-57.

Yano, O. \& Andrade-Lima, D. 1987. Briófitas do Nordeste brasileiro: Estado de Pernambuco. Revta. brasil. Bot., 10:171-181.

Yano, O. \& Mello, Z.R. 1992. Briófitas novas para o Estado de Roraima, Brasil. Acta Amazonica. 22(1):23-50.

Yano, O. \& Santos, S.X. 1993. Musgos da gruta de Mirassol, São Paulo. Acta Botanica Brasilica, 7(2):89106.

Yuzawa, Y. 1991. A monograph of subg. Chonanthelia of gen. Frullania (Hepaticae) of the world. Journ. Hattori Bot. Lab., 70:181-291. 